\title{
MASS LOSS IN SEMI-DETACHED BINARIES *
}

\author{
K. D. ABHYANKAR \\ Centre of Advanced Study in Astronomy, Osmania University, Hyderabad, India
}

(Received 7 June, 1983)

\begin{abstract}
Pre-Main-Sequence contracting objects, post-Main-Sequence expanding stars and mass-losing components of semi-detached systems all occupy more or less the same region in the conventional H-R-diagram. We make a transformation to variables $\Delta(\log L)$ and $\Delta\left(\log T_{e}\right)$, where $\Delta$ is the difference between the observed quantity, $\log L$ or $\log T_{e}$, and the value of that quantity which a star of the same mass would have on the empirical Main Sequence. It is demonstrated that a plot between the new variables clearly separates the mass-losing stars from other objects which is essentially an effect of the increasing abundance of helium relative to hydrogen.
\end{abstract}

\section{Introduction}

H-R-diagram is a powerful tool for studying the evolution of stars mainly because of the general validity of the Russell-Vogt theorem for stars in hydrostatic and thermal equilibrium. Given the total mass and run of chemical composition within the star, one can obtain a unique configuration with specific values of effective temperature and luminosity which fix the position of the star in the H-R-diagram. The zero-age Main Sequence is characterised by homogeneous chemical composition throughout the star and energy production by hydrogen burning in the core. Since it is possible to calculate the change of chemical composition caused by thermonuclear reactions one can trace the track of the star in the H-R-diagram as it evolves away from the Main Sequence. In general, the star expands and the effective temperature drops which causes it to move towards right into the giant and subgiant region of the H-R-diagram. There is also an increase in luminosity due to the increase in mean molecular weight caused by the conversion of hydrogen into helium in the central core.

The same region of giants and subgiants is also occupied by the pre-Main-Sequence contracting stars which are not in strict hydrostatic equilibrium. They are distinguished from the post-Main-Sequence objects by other characteristics such as: surrounding nebulosities, infrared excess, presence of emission lines in spectra, flare activity, and overabundance of lithium.

There is a third group of objects which also occupy the giant and subgiant region of the H-R-diagram, viz. the evolved components of semi-detached binaries (Kopal, 1956). It is now well-established that they have lost a large amount of their original mass, particularly from their hydrogen rich envelopes. As it is desirable to distinguish them from the other two groups we give here a method of doing so by means of a transformation of variables.

\footnotetext{
* Paper presented at the Lembang-Bamberg IAU Colloquium No. 80 on 'Double Stars: Physical Properties and Generic Relations', held at Bandung, Indonesia, 3-7 June, 1983.
} 


\section{The Choice of Variables}

The clue to the choice of variables comes from $\mathrm{R} \mathrm{CMa-systems} \mathrm{which} \mathrm{contain}$ secondaries of very low mass. In the case of $\mathrm{R} \mathrm{CMa}$ itself the evolved secondary has a mass of $0.2 M_{\odot}$ (Sahade, 1963; Radhakrishnan et al., 1983) which corresponds to a Main-Sequence star of spectral type M5 that would have a luminosity $3.5 \times 10^{-3} L_{\odot}$ and effective temperature of $2800 \mathrm{~K}$. However, its actual luminosity of $0.55 L_{\odot}$ and effective temperature of $4500 \mathrm{~K}$ make it appear like a Main-Sequence star of spectral type G8. Thus the secondary of R CMa is both overluminous and hotter for its mass; this is a characteristic of increased abundance of helium relative to hydrogen (cf. Schwarzschild, 1958; p. 143). Since the evolved components of semi-detached sytems are supposed to have lost a large portion of their hydrogen envelope exposing the inner

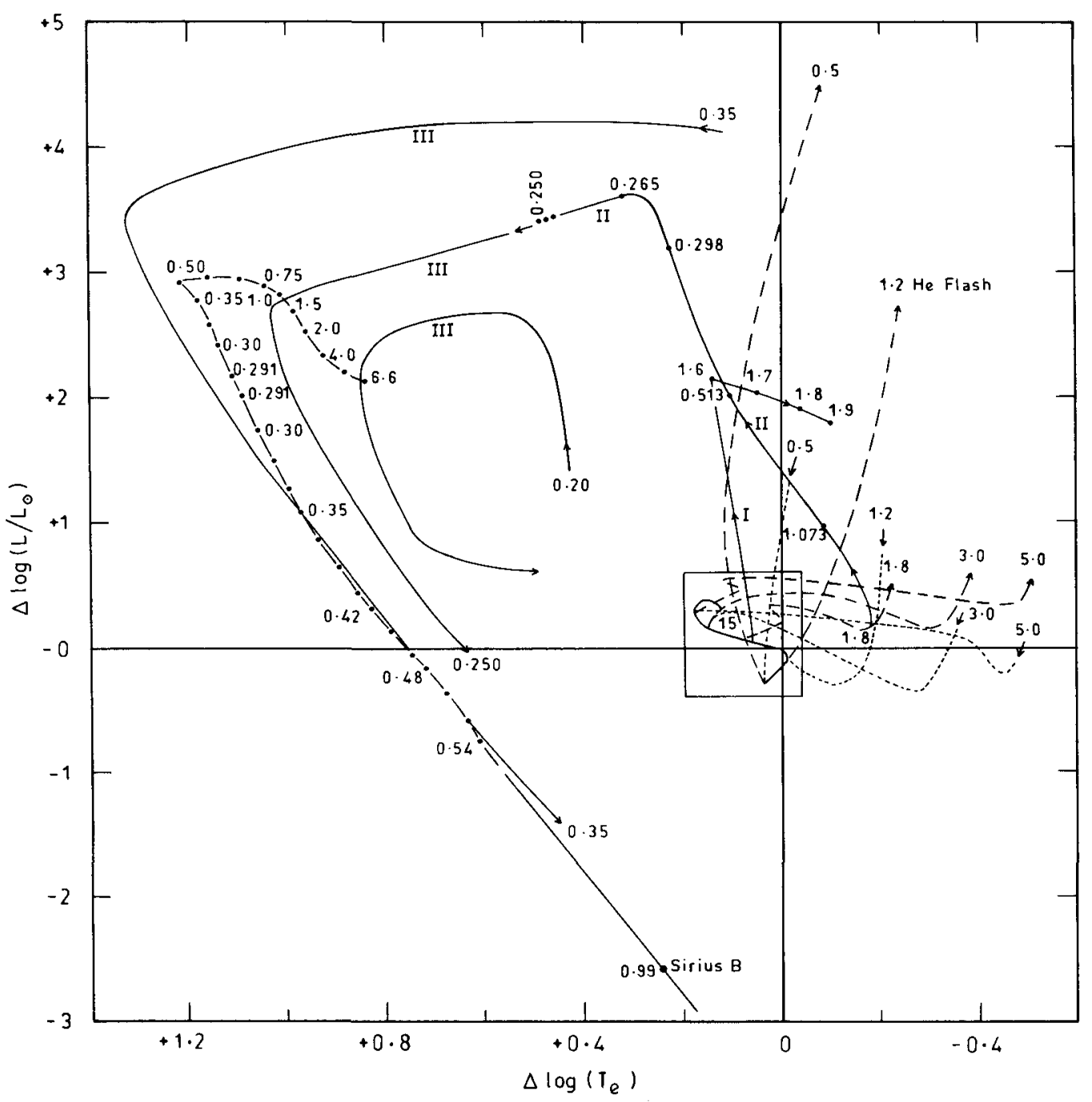

Fig. 1. Evolutionary tracks of various objects in the $\Delta \log L-\Delta \log T_{e}$ plot; see text for explanation. 
helium-rich core they should show both the increase in luminosity as well as effective temperature.

We choose, therefore, the following variables:

(i) $\Delta(\log L)=$ observed $\log L-\log L$ for a star of the same mass on the empirical Main Sequence.

(ii) $\Delta\left(\log T_{e}\right)=$ observed $\log T_{e}-\log T_{e}$ for a star of the same mass on the empirical Main Sequence.

Figure 1 shows the following groups of stars in the $\Delta(\log L)-\Delta\left(\log T_{e}\right)$ plane:

(a) Empirical Main Sequence is represented by just one point viz, the origin.

(b) The zero-age Main Sequence based on calculations of Iben (1965) for $X=0.708$, $Z=0.02$ is shown by the $S$-shaped curve near the origin. The difference from empirical Main Sequence arises because at the upper end it contains somewhat evolved stars while at the lowest end it contains stars which have just reached the zero-age Main Sequence. The square surrounding ZAMS is a measure of uncertainty in the estimation of our variables.

(c) Tracks (small dashed curves) of pre-Main-Sequence contracting single stars of various masses (marked on the curves) according to Iben (1965).

(d) Tracks (long-dashed curves) of post-Main-Sequence single stars of various masses (marked on the curves) due to Iben (1964).

(e) Track of a rapidly mass accreting star of mass $1.5 M_{\odot}$ in a binary (continuous curve marked I) according to Neo et al. (1977); the mass of the star at each stage is marked on the track.

(f) Track of the mass-losing component in a binary with original masses $M_{1}=1.8 M_{\odot}, M_{2}=0.7 M_{\odot}$ (continuous curve marked II) as calculated by Refsdal and Weigert (1969); here also the mass at various stages is marked on the track.

(g) Evolutionary tracks of helium white dwarfs in close binaries (continuous curves marked (III) according to Webbink (1975)); mass of the star is indicated on each curve.

(h) Helium Main Sequence (dash dot curve) due to Hansen et al. (1972); numbers of this curve give the mass of the helium star.

(i) A typical white dwarf, Sirius B, in the lower part.

It is seen that the pre-Main-Sequence contracting stars and post-Main-Sequence evolving stars both occupy a region to the right of the origin. The rapidly mass accreting component of a binary first moves slightly to the left due to the heating of the photosphere by the infalling material, but it also eventually moves to the right as the envelope grows in size. This track also shows that a star cannot accrete much of the mass lost by its companion. Most of the mass lost by the evolving component leaves the system through the outer Lagrangian points.

The track of the mass losing component is, however, quite distinct in that it moves up and to the left which corresponds to an increase in luminosity as well as surface temperature. This is obviously the effect of the decrease in the hydrogen content by mass loss from the envelope. The star eventually moves towards the helium Main Sequence and ultimately becomes a white dwarf. We thus see that the track of a mass-losing star in the $\Delta(\log L)-\Delta\left(\log T_{e}\right)$ plane is markedly different from those for pre-Main- 
Sequence and post-Main-Sequence single stars which have not lost mass during the course of evolution.

\section{Components of Semi-Detached Systems}

Figure 2 is an enlarged version of Figure 1 with components of semi-detached systems plotted therein. The open symbols represent the more massive primaries while the filled

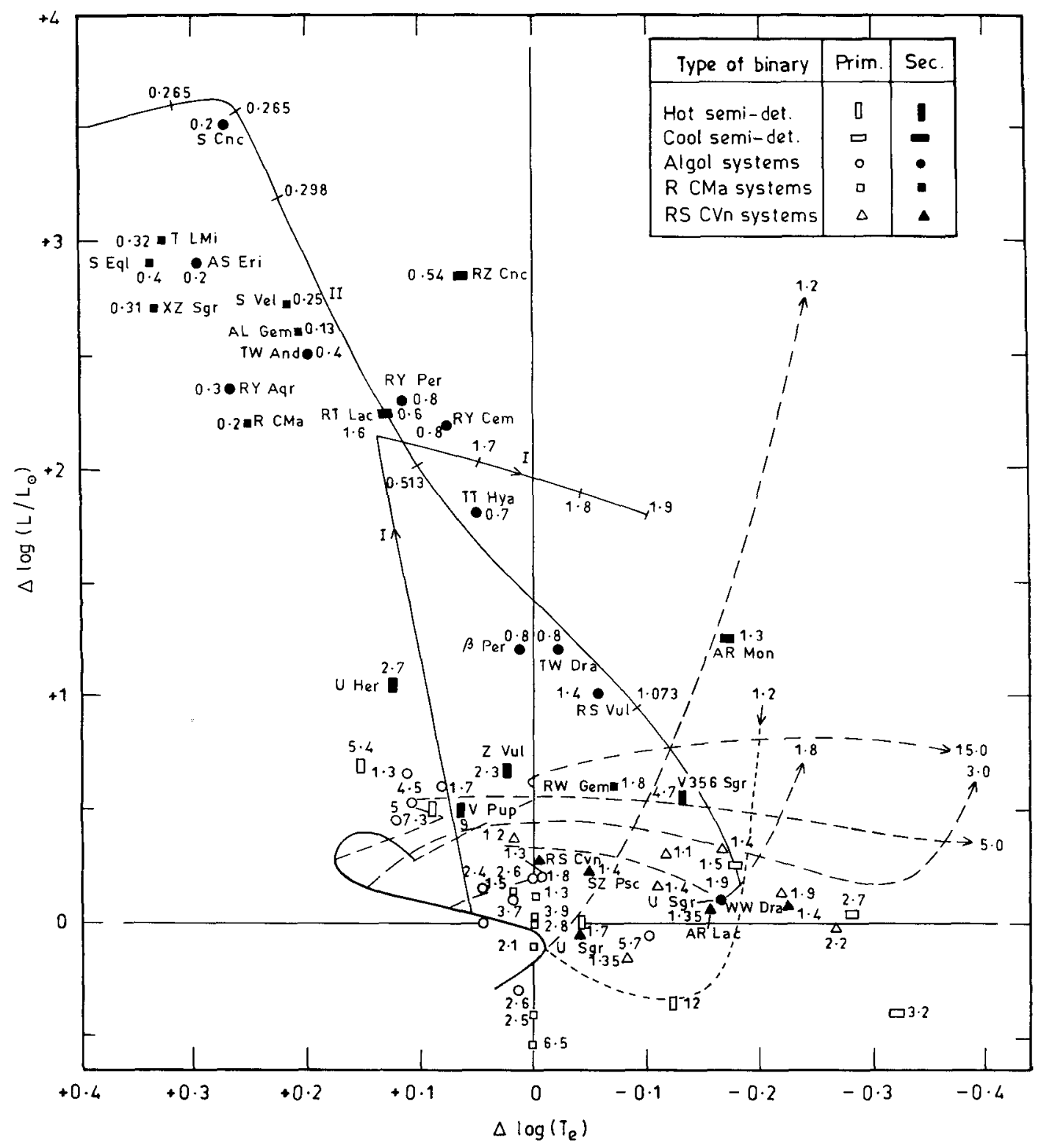

Fig. 2. Position of the components of semi-detached systems in the $\Delta \log L-\Delta \log T_{e}$ plot; see text for explanation. 
symbols denote the less massive secondaries; the numbers indicate masses of the two components. The following groups of binaries are shown in Figure 2:

(a) Hot semi-detached systems (vertical rectangles) listed by Popper (1980).

(b) Cool semi-detached systems (horizontal rectangles) listed by Popper (1980).

(c) Algol-systems (circles) listed by Popper (1980).

(d) R CMa-systems (squares) listed by Cester et al. (1979). The data for R CMa itself is ours (Radhakrishnan et al., 1983); in the case of other stars the more massive star was assumed to be a Main-Sequence star of observed spectral type for deriving the mass of the secondary.

(e) Six RS CVn-systems for which data are taken from Popper (1961, RS CVn), Arnold et al. (1979, SS Cam), Jakate et al. (1976, SZ Psc), Popper (1956, Z Her), Mardirossian et al. (1980; WW Dra) and Chambliss (1976; AR Lac).

It is clearly seen that the secondary components of most semi-detached systems occupy the region of mass losing stars in which the hydrogen content is considerably reduced. We would like to draw attention to TT Hya in which Kulkarni and Abhyankar (1981) had considered the secondary to be in pre-Main-Sequence contraction phase. From its position in Figure 2 it is clear that the star has definitely lost mass. Hence, its undersize nature which is confirmed by Kaul and Abhyankar (1982), has to be attributed to the second phase of contraction after considerable loss of mass from the envelope through the outer Lagrangian points by normal evolutionary process.

It may be noted that the secondaries of semi-detached systems invariably lie below the tracks of helium white dwarfs of same masses as calculated by Webbink (1975). This underluminosity indicates that they have lost mass so rapidly that the shell burning of hydrogen is also getting suppressed.

\section{Conclusions}

We have demonstrated that the $\Delta(\log L)-\Delta \log \left(T_{e}\right)$ plot separates the mass losing evolved components of semi-detached systems from the pre-Main-Sequence contracting stars and post-Main-Sequence expanding single stars. It is to be noted that only a small fraction of the mass lost by the evolved component can be accomodated on the companion. Hence, most of the mass is lost from the system through the outer Lagrangian points, and the more massive primary has essentially retained its original character with only a small increase in its mass. Since the evolved secondary must have been originally the more massive component the quantity $1-q$, where $q=m_{2} / m_{1}$ is the mass ratio, is a measure of the minimum fraction of mass lost by it. Hence, there should be a correlation between the mass ratio and the state of evolution of the secondary.

In order to find this correlation we consider the differential evolution of the two components of the semi-detached system in the $\Delta(\log L)-\Delta\left(\log T_{e}\right)$ plane. Defining $\delta=\Delta$ (secondary) $-\Delta$ (primary) we have plotted $\delta(\Delta \log L)$ vs $\delta\left(\Delta \log T_{e}\right)$ in Figure 3 for all the systems shown in Figure 2. We find a linear correlation (line $A B$ ) between these quantities such that systems with small value of $q$, which lie at the upper end of the line $A B$, show larger differential evolution by mass loss as compared to the systems 


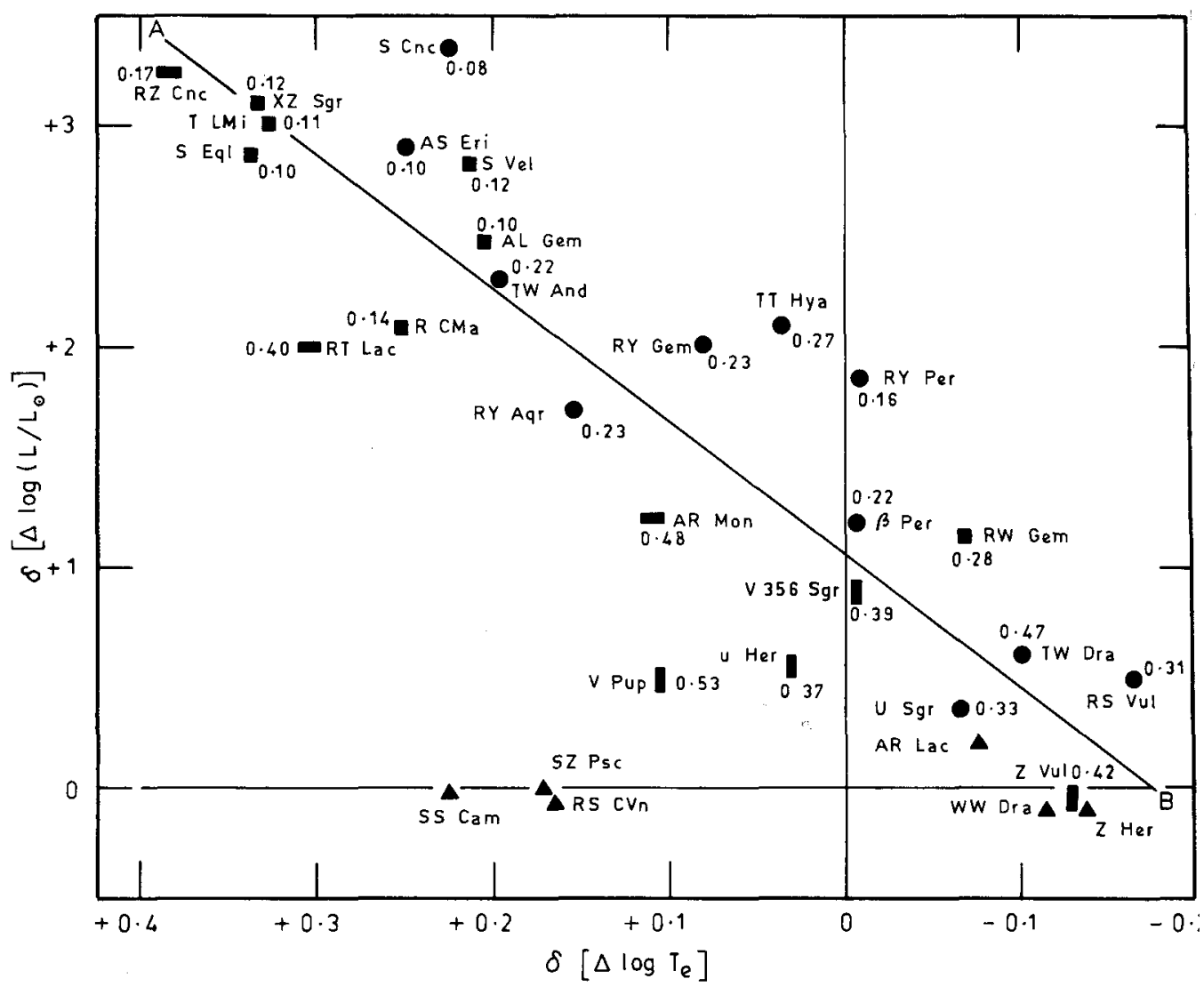

Fig. 3. Differential evolution of the components of the semi-detached systems; $q=m_{2} / m_{1}$ values are marked for each system.

with larger values of $q$ which lie at the lower end. The RS CVn-systems show the least differential evolution by mass loss and that also along the axis $\delta(\Delta \log L)=0$ as is expected from their almost equally massive detached components.

The correlation of overluminosity with $q$ was noted long ago by Struve (1954). We now find that the excess of effective temperature is also similarly correlated with $q$. As the evolved secondary loses mass with attendant reduction in the value of $q$, its hydrogen content is also reduced and the star becomes both overluminous and hotter for its residual mass.

\section{References}

Arnold, C. N., Hall, D. S., Montle, R. F., and Stuhlinger, T. W.: 1979, Acta Astron 29, 243.

Cester, B., Giuricin, G., Mardirossian, F., Mizzetti, M., and Milano, L.: 1979, Astron. Astrophys. Suppl. 36, 273.

Chambliss, C. R.: 1976, Publ. Astron. Soc. Pacific 88, 762.

Hansen, C. J., Cox, J. P., and Herz, M. A.: 1972, Astron. Astrophys. 19, 144. 
Iben, I., Jr.: 1964, Astrophys. J. 140, 1631.

Iben, I., Jr.: 1965, Astrophys. J. 142, 993.

Jakate, S., Bakos, G. A., Fernie, J. D., and Heard, J. F.: 1976, Astron. J. 81, 250.

Kaul, J. and Abhyankar, K. D.: 1982, J. Astrophys. Astron. 3, 93.

Kopal, Z.: 1956, Ann. Astrophys. 19, 298.

Kulkarni, A. G. and Abhyankar, K. D.: 1981, J. Astrophys. Astron. 2, 119.

Mardirossian, F., Mezzetti, M., Cester, B., and Giuricin, G.: 1980, Astron. Astrophys. Suppl. $39,73$.

Neo, S., Miyaji, S., and Nomoto, K.: 1977, Publ. Astron. Soc. Japan 29, 249.

Popper, D. M.: 1956, Astrophys. J. 124, 196.

Popper, D. M.: 1961, Astrophys. J. 133, 148.

Popper, D. M.: 1980, Ann. Rev. Astron. Astrophys. 18, 115.

Radhakrishnan, K. R., Sarma, M. B. K., and Abhyankar, K. D.: 1983, Astrophys. Space Sci. 99, 229 (this volume).

Refsdal, S. and Weight, A.: 1969, Astron. Astrophys. 1, 167.

Sahade, J.: 1963, Ann. Astrophys. 26, 80.

Schwarzschild, M.: 1958, Structure and Evolution of the Stars, Princeton University Press, Princeton.

Struve, O.: 1954, Mem. Soc. Roy. Liège 14, 236.

Webbink, R. F.: 1975, Monthly Notices Roy. Astron. Soc. 171, 555. 